Revista Arbitrada Interdisciplinaria KOINONIA

Año VI. Vol VI. N 12. Julio - Diciembre. 2021

Hecho el depósito de Ley: FA2016000010

ISSN: 2542-3088

FUNDACIÓN KOINONIA (F.K). Santa Ana de Coro. Venezuela.

María Belén Jarrín-Arias; Jorge Edwin Ormaza-Andrade

http://dx.doi.org/10.35381/r.k.v6i12.1296

\title{
Gestión de calidad para el sector de electrodoméstico
}

\section{Quality management for the household appliance sector}

\author{
María Belén Jarrín-Arias \\ maria.jarrin.36@est.ucacue.edu.ec \\ Universidad Católica de Cuenca, Cuenca \\ Ecuador \\ https://orcid.org/0000-0003-0900-9709 \\ Jorge Edwin Ormaza-Andrade \\ jormaza@ucacue.edu.ec \\ Universidad Católica de Cuenca, Cuenca \\ Ecuador \\ https://orcid.org/0000-0001-5449-1042
}

Recepción: 15 de marzo 2021

Revisado: 15 de mayo 2021

Aprobación: 15 de junio 2021

Publicación: 01 de julio 2021 


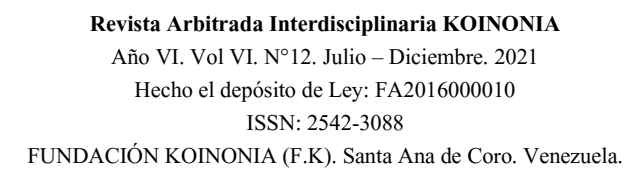

María Belén Jarrín-Arias; Jorge Edwin Ormaza-Andrade

\title{
RESUMEN
}

El objetivo es proponer la gestión de calidad para los almacenes de electrodomésticos en la ciudad de cuenca con la finalidad de mejorar la satisfacción del consumidor. Metodológicamente es de tipo descriptiva. la insatisfacción de los consumidores es debido a que no se informó adecuadamente antes de adquirir un servicio y/o producto, en un universo de 248 clientes, se realizó la encuesta a 183 clientes frecuentes, el 84,7\% afirma que ni tenían la información adecuada para adquirir un artefacto o la forma adecuada de utilizarlo. Se determinó que en las empresas de electrodomésticos deben profundizar en una información clara en el empleado ya que no cuentan con esa herramienta para llenar las expectativas del cliente, esto es debido a la rotación del personal en el área de ventas y a la demanda acelerada de modelos en producción.

Descriptores: Oferta y demanda; microeconomía; administración de empresas. (Palabras tomadas del Tesauro UNESCO).

\begin{abstract}
The objective is to propose quality management for electrical appliance warehouses in the city of Cuenca in order to improve consumer satisfaction. Methodologically it is descriptive. consumer dissatisfaction is due to the fact that they were not adequately informed before acquiring a service and / or product, in a universe of 248 clients, the survey was carried out on 183 frequent clients, $84.7 \%$ affirm that they did not even have the information appropriate to acquire an appliance or the proper way to use it. It was determined that appliance companies should delve into clear information on the employee since they do not have this tool to meet customer expectations, this is due to the turnover of staff in the sales area and the accelerated demand for models in production.
\end{abstract}

Descriptors: Supply and demand; microeconomics; business management. (Words taken from the UNESCO Thesaurus). 


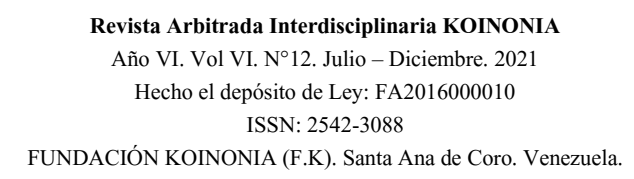

María Belén Jarrín-Arias; Jorge Edwin Ormaza-Andrade

\section{INTRODUCCIÓN}

Las ventas en general forman parte de nuestra vida diaria, considerado como un negocio totalmente antiguo pero eficiente, de tal manera que se manifiesta como un intercambio para cubrir necesidades de cada individuo llevando así a un abanico de opciones, las mismas que se manifiestan con un valor agregado caracterizado por la marca. Ahora bien, podemos detallar que existen diferentes marcas en el mercado y son las encargadas de producir a gran escala los productos para el consumo, al mismo tiempo son las responsables directas de cubrir las garantías emitidas por el fabricante bajo el régimen de la ley del consumidor, en el país la venta de electrodomésticos es representativa al ser productos de necesidad primaria en la vida de los ecuatorianos, se ha verificado que. En el Ecuador la industria de línea blanca comenzó a presentar en el año de 1964, dotando así de artefactos que facilitaran la vida de la población y, la primera compañía que se constituye se origina en la ciudad de cuenca que en ese entonces se denominaba Ecuatoriana de artefactos S.A. con su nombre más conocido hasta la fecha, Grupo Eljuri la prioridad que ellos definieron es la de crear cocinas ya que es el producto más utilizado, en las viviendas ecuatorianas, de manera consecutiva se constituye la siguiente compañía pero ya en la ciudad de Guayaquil, denominada Durex en el año 1964 específicamente el 4 de abril, tiene como función principal la elaboración de una línea de vasijas pero en material denominado hierro enlozado pasando a ser el primero en elaborar este tipo de utensilios para el hogar (Bonilla-Rivera \& Paraguay-Alvarado, 2017). Cabe considerar en otra parte que las tendencias en ventas cambiaron por el aspecto de la pandemia.

Debe señalarse que es imposible negar el impacto sobre la economía que nos afecta hasta la fecha actual con respecto a la pandemia que tuvo mayor auge en marzo del 2020 y que es comúnmente llamado COVID-19. Especialmente en él cierre total de algunos negocios, él no pago de deudas adquiridas para incrementar productos que generen ganancias a micro empresarios y los cambios en el comportamiento de los consumidores que, al verse paralizados en su economía, implementaron un cambio radical, de esta 
forma las empresas como negocios pequeños han tenido que adaptarse a la circunstancia adversa para salvaguardar parte de sus bienes (Bodnar, 2020). Cabe recalcar que las empresas dotan de capacitaciones, charlas y talleres frecuentemente a su grupo de empleados para mejorar y satisfacer las necesidades del consumidor profundizando en el servicio a sus clientes. En efecto, el objetivo es proponer la gestión de calidad para los almacenes de electrodomésticos en la ciudad de cuenca con la finalidad de mejorar la satisfacción del consumidor.

\section{Referencial Teórico}

\section{Insatisfacción del consumidor en la gestión de calidad}

En este sentido, (ISO 9001, 2020) afirma: "La gestión de la calidad establece que el 90\% de los defectos o problemas de calidad son generados por los propios procesos y no por el personal". Por lo tanto, el anuncio del consumidor es importante analizar un intercambio de posiciones, puesto si fuéramos el cliente nos gustaría una actitud buena y positiva frente al problema que nuestra versión sea escuchada con la finalidad de que el sustento sea un reclamo adecuado, sin embargo el cliente en el mayor de los casos buscara la devolución del dinero, si se trata de un servicio, o el cambio inmediato si es cuestión de un producto, satisfacer al cliente convertirá de esta forma una fortaleza que simbolizara a nuestra marca (Delgado-Estrada, et al. 2018).

Por supuesto que este fenómeno va a depender de muchos factores más allá de los económicos, la insatisfacción del cliente puede llegar a tocar puntos muy específicos como a continuación presentamos: social, sentimentales, espiritual, basado en creencias o costumbres. Resulta así mismo interesante que los reclamos de los consumidores por la falta de calidad nos conlleva a un punto muy relevante, es el de utilizar estos contratiempos para ir desarrollando un enriquecimiento con nuestro trato o servicio.

Creo que es evidente, el cliente al hacer un llamado de atención, alerta de que estamos desviándonos del camino correcto, de una prestación de servicios adecuados, a pesar de verlo como algo negativo, esto fortalece a la empresa y pone en alerta las fallas que 


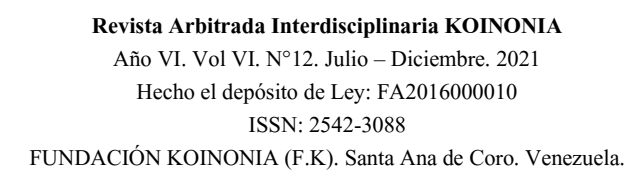

María Belén Jarrín-Arias; Jorge Edwin Ormaza-Andrade

a futuro desembocaran a una pérdida de clientes. Al comparar estas evidencias, es interesante que reclamar por parte del cliente, se debe tomar como una oportunidad para seguir mejorando, más no como una amenaza ya que los errores no siempre suelen ser percibidos por el personal.

Las empresas se encuentran convencidas que mantiene contentos a los clientes, pero no se puede concretar esta versión, al contrario de considera lo siguiente, si los clientes solo se mantienen cautivos, porque desvincularse tal vez genere otro tipo de inconvenientes, Porque en el mercado las alternativas son iguales, por ende mantener una fidelidad con su prestador por el momento será lo más adecuado hasta encontrar otras opciones, Porque el valor económico es considerable a pesar de no contar con una buena calidad en todo caso, lo aquí detallado hace referencia a que el prestador tiene que cambiar la visión que hasta la fecha ha realizado sobre su gestión de calidad, lo ideal es recalcular a corto plazo para ir detectando fallas en la actualidad y las mismas nos terminen afectando los ingresos de su negocio (Palmira-Lopez, 2010).

En efecto las empresas cuentan con un departamento de post venta, encargado de registrar y resolver los reclamos o garantías dirigidas, otra tarea prioritaria es calcular los indicadores de ratios que más utilizamos. Mediante una medición de ratios se llevará un control adecuado de los reclamos, pues depende de la empresa la manera de registrarlos, para eso se necesita hacer un corte semanal o mensual dependiendo de la afluencia de clientes, a continuación, se presenta 2 tipos de ratios más comunes que son:

Ratio de quejas atendidas: El dato establece que la medición de reclamos atendidas sobre el total de reclamos recibidas nos dará un resultado, si las mismas fueron atendidas en su totalidad; $100 \%$.

Ratios de quejas atendidas

Cantidad de quejas atendidas

Cantidad de quejas recibidas

$\times 100$ 


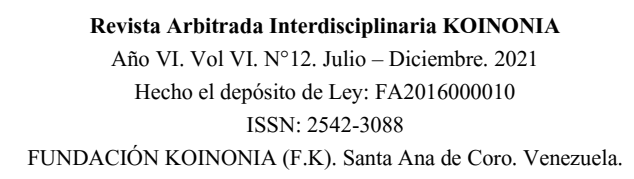

María Belén Jarrín-Arias; Jorge Edwin Ormaza-Andrade

Pero en el detalle expuesto no refleja que la queja se haya resuelto, solo la atención sin conocimiento de que el resultado sea el esperado, para eso exponemos una nueva ratio. Ratio de quejas resueltas: El detalle expuesto, establece que la medición de quejas resueltas por un total de quejas recibidas, demostrara el resultado adquirido.

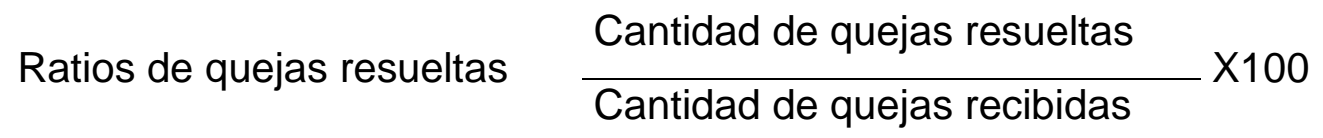

Dicho de otro modo, lo aquí detallado, nos arroja información sobre la medición de eficiencia de la empresa al resolver los reclamos inscritos. Retomando la idea inicial, en conclusión, los registros por la insatisfacción del consumidor en la gestión de calidad, termina siendo un llamado de atención, pero a nuestro favor faltaría de allí, resolverlos de la manera más correcta (Silva-Treviño, et al. 2021).

Por otro lado, un inadecuado comportamiento por parte del personal, se debe considerar que esta causa va de la mano con una buena educación y, como punto primordial la paciencia. De esta manera se ha estudiado las causas que perjudican a la empresa pero que sin su presencia no sería fácil percibir fallos para mejorar continuamente.

\section{Análisis de la gestión de calidad en el mercado}

En la actualidad la gestión de calidad es el punto de partida que toda empresa ya sea de servicios o productos, la misma que debe tener como prioridad en la identificación de su nombre o marca que deben ser analizadas e implementadas. En resumidas cuentas, la gestión de calidad, abarca varios procedimientos que llevan a un propósito que es el de tratar de ni cometer errores y que el número sea reducido, con la finalidad que los productos tengan un valor agregado que no se identifique en el momento que se ha producido sino de evitarlos en su mayoría, estar allí donde Interviene la organización sobre la gestión de calidad para procurar no incrementar el número de fallos, garantizando la calidad en el producto final, sino que también en el 


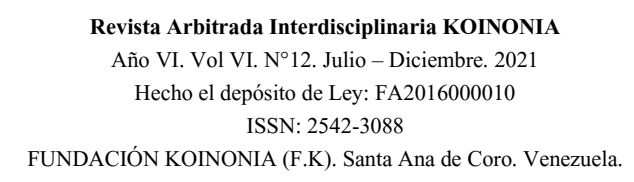

María Belén Jarrín-Arias; Jorge Edwin Ormaza-Andrade

proceso que conlleva la prestación del mismo (ISO 9001, 2020). Dicho de otro modo, la gestión de calidad son procedimientos meticulosos que se llevan a cabo en la prestación del servicio (Alcívar-Arízaga, et al. 2019), en consideración se necesitan conocer los detalles para que, o a que va enfocada un análisis sobre los procesos de gestión de calidad dentro del mercado.

Esto nos direcciona al mejoramiento esencial, aplicar nuevos procesos que a la fecha carezca o que se encuentren con fallas, debemos saber que al ofrecer un producto no solo es la parte física ni el resultado de un servicio, luego del análisis, en el camino se debe ir implementando procesos para la creación y aplicación de un buen sistema de gestión de calidad, adaptables a las necesidades que estén dirigidas mediante una estructura que lo llevara a definir claramente las exigencias de los consumidores (Antúnez-Saiz, 2016).

Es importante detallar que sin el análisis previo no se detectaran puntos claves para un mejoramiento de la calidad en el mercado. A continuación, detallaremos los análisis más relevantes que podemos implementar en el mercado para obtener mejores modelos enfocadas en la gestión de calidad.

El ciclo de mejora PDCA: El concepto de sus siglas, planificar, hacer, verificar y actuar, denominado círculo de Deming por su creador Edwards Deming, donde abarca, que el mejoramiento debe continuar, siguiendo estos 4 métodos enfocados en la calidad que consta en hacer descender los fallos que se vuelvan minoritarios y ascender la eficiencia de prestación de servicios con total eficacia, la composición de estos ciclos deben ser reanudados de manera que cuando culmine la etapa final este vuelva a la primera, al iniciar el proceso nuevamente, se detectaran los fallos estas formas harán que las actividades sean evaluadas periódicamente para incorporar nuevas mejoras (MonteroTapia, 2019). Algunas de sus manifestaciones indican que cada sigla es meticulosamente estudiada, por ende, veremos a continuación su significado:

Planificación: visualiza un punto analiza e identifica su problema, de allí queda establecer metas y actividades para enfocarse en emprender nuevos mecanismos para mejorar 
Desarrollar: comienza el desarrollo de las actividades para ejecutar o desarrollar las actividades propuestas, es indispensable que cuando se comience sea importante un modelo más pequeño y manejable hasta ir incorporando otros procesos más extensos Comprobar: esta etapa es netamente de verificación donde se confirma el resultado de las actividades propuestas en el desarrollo si el proceso fue o no el adecuado servirá Actúa: finalmente se aplica el resultado que se obtiene en todo este proceso visualizando así las mejoras y los objetivos obtenidos (Bernal, 2013).

Excelencia en la gestión Malcolm Baldrige: El siguiente modelo se encuentra definido como excelencia, en este concepto detalla un sin número de probabilidades enfocadas a todo tipo de empresas basándose en una lista de puntos relevantes que colaboran a un análisis más profundo y específico.

Liderazgo visionario: generalmente, es responsabilidad de la parte de la gerencia que este orientado hacia necesidades del cliente, garantizar, planificar y desarrollar un plan estratégico fundamentado.

Excelencia impulsada por el cliente: es importante analizar los gustos o preferencia de los clientes para que a futuro adelantarnos a sus necesidades y que nuestras ofertas ya se encuentren predestinadas.

Aprendizaje organizacional y personal: como su nombre lo indica, el aprendizaje continúa, preparación, actualización y prioridad en las habilidades existentes en el personal, a las inversiones internas o externas.

Agilidad: predomina la rapidez y eficiencia para mejorar los tiempos en la productividad, innovación, implementación es un factor indispensable para mejorar el servicio enfocado al éxito.

Orientación al futuro: planificación y desarrollos estratégicos que generen beneficios futuros.

Gestión para la innovación: al completar cambios significativos, crear innovaciones con frecuencia generando diversidad en la gestión de calidad llama la atención a clientes potenciales 


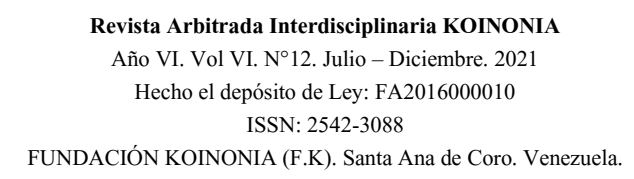

María Belén Jarrín-Arias; Jorge Edwin Ormaza-Andrade

Gestión basada en hechos: se refiere a los resultados obtenidos, respaldos específicos Responsabilidad social: basada en la conciencia sobre disminuir el impacto en el futuro. Orientación a la obtención de resultados y la creación de valor: los valores de desempeño en el personal detectaran una base específica de resultados obtenidos

Perspectiva de sistema: obtener resultados específicos del programa (Baldrige National Quality Program USA, 2006).

Modelo de Excelencia EFQM: Fue creada en 1988 fundación europea para la gestión de la calidad es una organización sin fines de lucro basadas en un modelo de calidad y con una misión en excelencia, luego en 1999 cambio de nombre pasando a ser modelo de excelencia 2000 a sus inicios fue compuesta por 14 empresas europeas, la finalidad de esta organización es la orientación de resultados que garanticen un servicio con éxito se adecua a cualquier tipo de empresa u organización sin importar tamaño o tiempo en el mercado, está compuesta por 9 criterios divididos en 5 facilitadores son las personas encargadas de liderar políticas y estrategias planifican alianzas enfocadas en los recursos todo esto conlleva a procesos que desembocan en los resultados siguientes: personas, cliente y sociedad obteniendo así efectos claves del modelo EFQM (Martínez, 2008).

Con lo anteriormente detallado podemos hacer un plan de trabajo para mejorar la gestión de calidad en la empresa asignada, se puede elegir uno de estos 3 modelos, el que más se adapte al perfil de su empresa, de tal forma que el primer paso es incorporar un análisis para detectar el problema tanto en producción o servicios seguidos de este punto se incorporara charlas, capacitaciones cursos intensivos y en especial que el personal se comprometa de antemano a ser responsable y trabajar de manera adecuada, se analizara y un plan estratégico para visualizar a futuro los fallos posibles sin dejar que estos se acumulen, de esta manera será más provechosas y menos riguroso establecer nuevas reglas para llevar a cabo la gestión de calidad y, por último se incorporara la metodología adecuada para implementar en un futuro de servicios con excelencia en la calidad. 


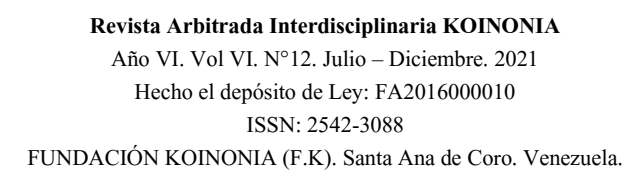

María Belén Jarrín-Arias; Jorge Edwin Ormaza-Andrade

\section{Procesos y herramientas para la mejora continua de la gestión de calidad}

Si nos basamos en que corregir, armar o diseñar una ruta hacia la gestión de calidad enfocados en que los establecimientos, obtenga una filosofía en la prestación de servicios, lo ideal es proponer una nueva cultura e incentivar al personal a trabajar en equipo, podríamos hablar ya de un proceso establecido y adjuntar algunas herramientas adecuadas para mejorar continuamente en el servicio. De esta manera los procesos a llevar serán ya conceptuados ni realizados por el impulso, por la facilidad de palabra o por experiencia en resolver algunas situaciones complicadas o comunes que se dan al día más bien se necesita tener ya descritas las herramientas o procesos que mejoraran el servicio al cliente (León-Ramentol, et al. 2018).

Por lo tanto, entendemos que dichas herramientas o proceso son muy significativos e indispensables para salvaguardar el bienestar de los clientes. Cabe recalcar, de esta manera se podría definir como técnicas o procedimientos que se presentan como funciones y que tiene la finalidad de ayudar a las empresas con la medición en la calidad de los servicios, esto nos llevara a obtener mejores resultados y resolver los problemas aplicando estas herramientas de manera adecuada para mejorar la calidad, ellas nos permiten generar nuevas técnicas, desarrollar, establecer y, más que nada plantear tareas con la finalidad de garantizar los objetivos determinados por cada institución e ir implementando nuevos métodos por el personal que es el motor de una empresa y que se encuentre comprometido en desarrollar un mejor servicio (Medina-León, et al. 2019). Por último, es conveniente detallar, algunas de las herramientas más utilizadas por las instituciones para manejar adecuadamente el registro de calidad destacando las siguientes.

Diagrama de causa y efecto o diagrama de ishikawa: Mantiene el nombre por su creador Kaoru Ishikawa es conocido como diagrama de espina de pescado por la forma como se representa gráficamente, en este diagrama se puede identificar las causas específicas del porque son desfavorables los resultados para la empresa, estas causas van 


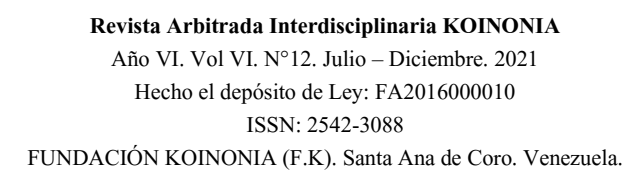

María Belén Jarrín-Arias; Jorge Edwin Ormaza-Andrade

enraizadas desde sus inicios sin ser detectadas, ocasionando el efecto donde radica el problema, también es favorable esta herramienta porque visualiza el entorno donde se pueden encontrar los posibles factores que a futuro ocasionaran problemas.

De la misma manera presenta la oportunidad de solucionar o mitigar el efecto, claro de las causas se desmiembran las sub causas, como ejemplo exponemos si el personal no es apto para las funciones encomendadas es tal ves debido a que no tiene experiencia en ese cargo ni se encuentra conforme en el lugar que le asignaron. Es recomendable para que funcione la técnica de manera correcta es precisa dividirlas por categorías 0 funciones como:

Máquinas: es indispensable que las máquinas a utilizar ni se encuentren defectuosos ni obsoletos o que carezcan de los mismos.

Métodos a utilizar: la metodología en algunos casos es inapropiada, los tiempos no establecidos favorecen para presentar resultados, la presión sobre el personal no es la recomendable.

Materiales: son las herramientas principales para el trabajo por ende se debe tener un stock en repuestos o accesorios.

Personal: al ser sujeto de nuestro estudio se puede verificar si tiene o no tiene ninguna experiencia, si se encuentra comprometido en llevar sus funciones de la manera correcta. Sobre las bases de las ideas expuestas, y los procesos detallados del presente estudio depende de cada empresa tomar la decisión de aplicarla o no, tomando en cuenta que es muy favorable esta herramienta que desglosa las causas donde se genera el problema como resultado final (Romero-Bermúdez \& Díaz-Camacho, 2010).

Diagrama de pareto: Es relacionado con un análisis llamado la curva 80/20 que se define de la siguiente forma si un producto o servicio presenta fallas es el $80 \%$ y, las causas que lo llevaron a ese desenlace pasarían a ser el 20\%, evidentemente las causas así sean mínimas agrandan en proporción el problema, se representa por medio de una gráfica combinada que fue creada por Wilfredo Pareto, su estudio también en conocido como análisis de pareto, se lo denominó así cuando identifico que las riquezas en gran 
proporción era el $20 \%$ de la población mientas que el $80 \%$ ni obtenía remuneraciones prudentes, de esta manera se especifica la frecuencia que ocurren los problemas, así también detalla la importancia que representan los mismos, para trazar un diagrama de pareto es indispensable obtener la información sobre el tema a tratar o analizar (BonetBorjas, 2005). En otras palabras, una vez recopilada la información se tiene que tabular los datos, para que sea entendible se presenta un ejemplo sobre la reincidencia de fallo al mes en línea café.

Para tal efecto, primer paso obtener un listado de productos con la información de cuantas veces al mes esa marca se siniestra, en la frecuencia van los datos de siniestro y se ordena de mayor a menor, el porcentaje se saca dividiendo el número por el total de la frecuencia y se presenta el valor en porcentaje, el acumulado, se coloca la frecuencia del primer producto luego en la siguiente celda se coloca igual el acumulado de la primera celda más la frecuencia del siguiente producto para sacar los valores del porcentaje acumulado se coloca la primera celda de la frecuencia acumulada y se divide para el total representado por un valor con porcentaje quedando de esta forma la tabulación de los datos para poder trazar la gráfica de una tabla cumulada.

Siniestrabilidad en línea café

$\begin{array}{lcccc}\text { Productos } & \text { frecuencia } & \% & \text { acumulado } & \% \text { acumulado } \\ \text { Led global 32 } & 12 & 23 \% & 12 & 23 \% \\ \text { Led doppio 49 } & 10 & 19 \% & 22 & 42 \% \\ \text { Led indurama 43 } & 9 & 17 \% & 31 & 60 \% \\ \text { Led samsung 32 } & 8 & 15 \% & 39 & 75 \%\end{array}$

Diagrama de flujo: Es una representación gráfica donde determinan los pasos a seguir de un algoritmo representado por figuras entrelazadas entre sí, de esta manera se desmiembra lo más elemental sobre el tema a tratar, la aplicación de este diagrama puede ser utilizada en general a todos los procesos o empresas donde sea necesario 
establecer información para mejorar la cálida de la gestión, cabe recalcar que las formas más utilizadas es la horizontal y vertical que es donde se van a direccionar los símbolos que tienen funciones específicas para poder interpretar la información recolectada y son las siguientes símbolos:

Ovulo: representa el inicio o el final del diagrama

Rectángulo: detalla las actividades

Rombo: Es el que toma las directrices a seguir, después de responder una pregunta

Flechas: es utilizada para entrelazar la información

Símbolos: define las actividades en el proceso

A través de las figuras se representa gráficamente el problema, por medio del cual se identifica la calidad que demande la empresa, mejorando la necesidad del cliente y acercándose a sus expectativas, de esta manera la comunicación se mejorará y los tiempos se verán reducidos a beneficio de la empresa. Es indispensable que, su equipo de administración se mantenga pendiente en manejarlos adecuadamente y no caer en el descuido, llevando así a reducir sus efectos, los mismos que solo le llevaran empezar de nuevo con la implementación de otro tipo de herramientas.

\section{MÉTODO}

Se desarrolló una investigación desde una fundamentación metodológica descriptiva con diseño no experimental, empleandose los clientes externos de los almacenes de electrodomésticos de la ciudad de cuenca, la mayoría de los clientes son frecuentes y con créditos activos se utilizó el tratamiento muestral con la siguiente formula:

$$
\begin{array}{|c}
\mathrm{N}^{*} Z 2{ }^{*} \mathrm{p}^{*} \mathrm{q} \\
\mathrm{d} 2^{*}(\mathrm{~N}-1)+Z 2^{*} \mathrm{p}^{*} \mathrm{a}
\end{array}
$$

$\mathrm{N}=$ Número total de la población o universo

$Z=$ Coeficiente de seguridad, seleccione el coeficiente de seguridad de acuerdo con el margen de error 
Z2= elevar Z al cuadrado (multiplicar el valor por sí mismo)

$p=$ proporción esperada, es valor fijo

$q=1-p(1-0,05=0,95)$

$\mathrm{d}=$ precisión $=0,1$ (valor fijo)

$\mathrm{d} 2=$ Elevar $\mathrm{d}$ al cuadrado (multiplicar el valor por sí mismo)

$p^{*} q=$ Multiplicar el valor de $p$ por el valor de $q$

Para mejor interpretación de las encuestas se calcula la muestra, obteniendo de un universo de 248 clientes de varios almacenes y, se aplicará la encuesta directamente a 183 clientes frecuentes y que a la fecha actual se encuentran con créditos activos.

De ese modo, se aplicó encuesta online y cuestionario con la finalidad de recopilar datos, los cuales fueron procesados mediante estadística descriptiva, siendo presentados en porcentajes y frecuencias en relación a describir los indicadores de la variable de investigación.

\section{RESULTADOS}

Cabe considerar que los resultados obtenidos sobre la gestión de calidad en los almacenes de electrodomésticos en la ciudad de la cuenca, refleja la insatisfacción de los clientes en el momento de adquirir un servicio o un producto, recopilando así información valiosa que contribuirá con esta investigación.

\section{Insatisfacción en el consumidor}

Podríamos resumir continuación que la insatisfacción de los consumidores es debido a que no se informó adecuadamente antes de adquirir un servicio y/o producto, en un universo de 248 clientes, se realizó la encuesta a 183 clientes frecuentes, el 84,7\% afirma que ni tenían la información adecuada para adquirir un artefacto o la forma adecuada de utilizarlo, quedando solo el $22.4 \%$, que afirma a ver investigando las características principales, como también sus ventajas y desventajas al adquirir un producto. 


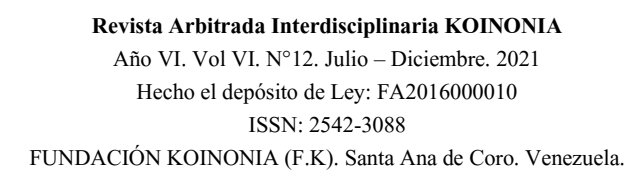

María Belén Jarrín-Arias; Jorge Edwin Ormaza-Andrade

Sin embargo, existe el 59\% de clientes que manifiestan que la información dotada específicamente en el momento de la compra por parte del personal sigue careciendo de veracidad, o que los conocimientos del colaborador ni son específicos para cubrir la expectativa del manejo adecuado del producto o servicio, sin embargo existe una contra parte que manifiesta lo siguiente que el $41 \%$ de clientes afirman que con personal capacitado, si les proporciona información adecuada en el momento de cerrar una venta como las características principales para la obtención de un producto, de esta manera queda acentuado que es indispensable y necesario obtener la información primaria antes de la compra.

\section{Productos o servicios inadecuados}

En la obtención de un servicio o producto el cliente se enfoca, en que los resultados sean satisfactorios, cabe recalcar si se trata de un bien generalmente viene de la mano con una garantía por un tiempo específico, bajo las características y reglamentos definidos ya sea por una marca o el proveedor, y si el caso es un servicio la garantía va relacionada con el tiempo que dure dicho servicio, de esta manera, en la encuesta realizada al universo de 248 clientes el $82.5 \%$ ha presentado alguna vez una aqueja por un servicio o un producto inadecuado, según la encuesta se confirma que el $54.7 \%$ son específicamente por un mal servicio el mismo que procede con un reclamo en el momento preciso que falle la prestación, de la misma forma se detalló en las encuestas realizadas el $42,6 \%$ es representado con fallas en un producto, puede estar manifestado con varios días posteriores a la compra.

Para cubrir la garantía el producto debe presentar daños de fábrica más no como daño provocado por el cliente, basándose en la información obtenida un porcentaje de 78,1\% siendo el más alto han presentado una queja por un producto con falla de 0 a 5 veces, bajando así el porcentaje se presenta que tan solo el 13,1\% presenta problemas de 6 a 10 veces y con un porcentaje minino el $8,7 \%$ de 11 a 15 veces ha inscripto un reclamo, 
pero en los resultados obtenidos se puede obtener que tan solo el $17,5 \%$ nunca ha presentado ningún tipo de reclamo.

\section{Tiempo de solución}

El variante tiempo de solución, es lo que caracteriza al proveedor o a la marca responsable de cubrir la garantía, dotando de solución al problema manifestado, mediante la información obtenida en las encuestas detalla lo siguiente.

Los datos sobre el tiempo de solución que muestran en las encuestas es que el $47,5 \%$ dura de 1 a 15 días en cubrir la garantía, bajando así el porcentaje, pero elevando los días de solución el $29,5 \%$ va de 16 a 30 días en espera y que el $23 \%$ dura más allá de los 31 días en recibir una respuesta por parte de proveedor de servicios, específicamente si se trata de un producto el mismo que termina con la devolución de dinero o cambio, en un porcentaje de $47 \%$, pero adicional a este resultado se manifiesta que el $53 \%$ recibe el mismo producto con las adecuaciones necesarias y cambio de repuestos originales en su producto inicial.

\section{Atención al cliente}

La finalidad de esta investigación está relacionada netamente a la atención al cliente con las herramientas adecuadas como son información y buen trato, podríamos resumir a continuación que, la amabilidad y la atención del agente de servicios que recepto su requerimiento, tiene el $12,6 \%$ de porcentaje representado como malo dentro de 183 cliente encuestados, y se representa el $73,2 \%$ como regular siendo el porcentaje más alto, pero con una calificación negativa para una gestión de calidad adecuada, de ahí refleja como excelente solo el $14,2 \%$, de esta manera se implementa un sistema de gestión de calidad, donde la parte administrativa involucre en todos los aspectos.

Al personal encargado de brindar un buen servicio al cliente, llevara a mejorar la imagen de la empresa mediante el diseño de nuevas estrategias, lineamientos y planificación de políticas para los procesos de servicios, adicional se deberá contar con una medición 
donde se pueda dar seguimiento de los objetivos planteados, según los resultados son 2 las partes más preponderantes donde se debe enfatizaren el servicio y son los siguientes, la atención al cliente y el chequeo de los productos ofertados (Perez-Montero, 2013).

\section{PROPUESTA}

El presente trabajo describe una propuesta para un sistema de gestión de calidad basado en una encuesta realizada a los clientes de los almacenes de electrodomésticos de la ciudad de cuenca, la misma que se divide en 4 procesos iniciando desde un análisis situacional de la empresa se procederá a establecer estrategias para ser aplicadas y posterior se dará un mejoramiento continúa.

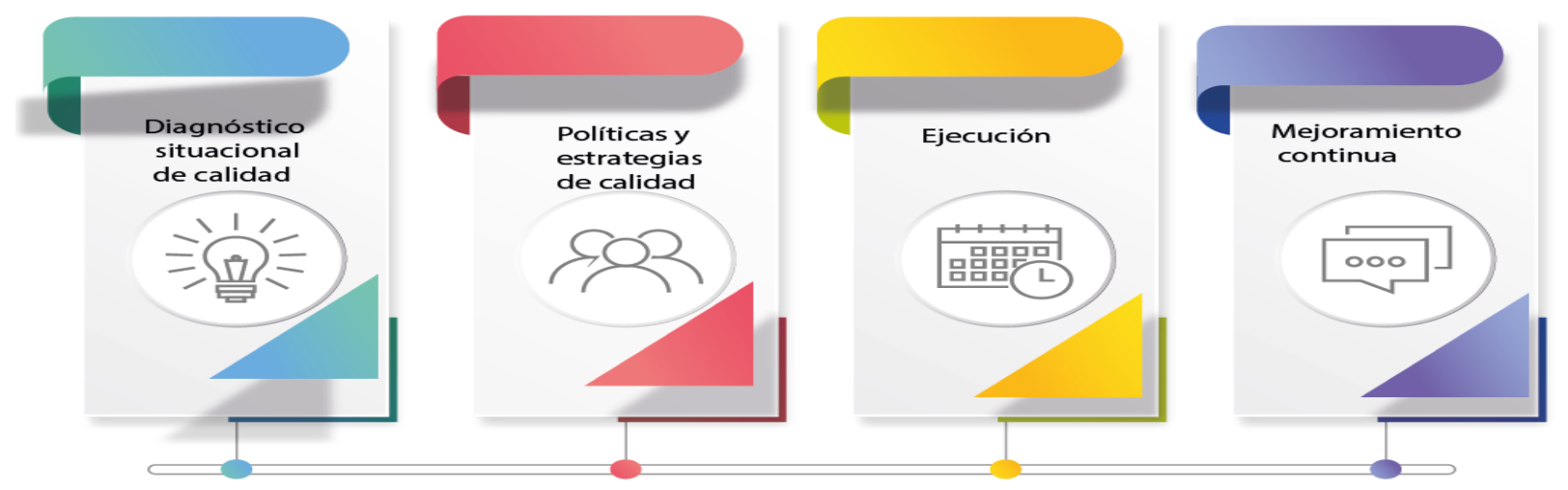

Figura 1. Estructura de la propuesta de investigación. Elaboración: Los autores.

\section{Diagnostico situacional de calidad}

Mediante la matriz FODA se realizó un análisis de los aspectos internos y externos de las empresas de venta de electrodomésticos, se puede determinar que en los factores internos la debilidad toma la ventaja por la devolución de producto, llevando así a una anulación de la venta por ni cumplir las expectativas del cliente que generalmente son por equipos de calidad baja o tecnología antigua, adicional a este se determina que no existe una actualización en el equipamiento para exhibir los productos de una manera correcta y que atraiga al consumidor, estos parámetros determinan una debilidad baja en 
el análisis, incrementando la amenaza las mismas que se enfocan en la competitividad del mercado y el incremento de productos sustitutivos con bajos precios lo cual opaca la venta de algunas casas comerciales que no van acorde a la actualización del producto y servicio de esta forma se determinan las partes negativas y positivas, puntos que se va a considerar en la propuesta, se adjunta la tabla de los factores internos, externos y la ponderación correspondiente al análisis realizado.

Tabla 2.

Análisis FODA.

\begin{tabular}{|c|c|c|c|c|}
\hline & \begin{tabular}{|l|} 
Factores \\
internos
\end{tabular} & Ponderación & Clasificación & Valor \\
\hline \multicolumn{5}{|l|}{ Fortalezas } \\
\hline 1. & $\begin{array}{l}\text { Estabilidad } \\
\text { laboral }\end{array}$ & $10 \%$ & 3 & 0,3 \\
\hline 2. & $\begin{array}{l}\text { Calidad de } \\
\text { productos }\end{array}$ & $20 \%$ & 4 & 0,8 \\
\hline 3. & \begin{tabular}{|l|} 
Crédito con \\
proveedores
\end{tabular} & $10 \%$ & 4 & 0,4 \\
\hline 4. & \begin{tabular}{|l} 
Empleados \\
comprometidos \\
\end{tabular} & $5 \%$ & 3 & 0,15 \\
\hline 5. & $\begin{array}{l}\text { Cartera de } \\
\text { clientes }\end{array}$ & $5 \%$ & 4 & 0,2 \\
\hline Debilidades & & & & 0 \\
\hline 1. & $\begin{array}{l}\text { Recuperación } \\
\text { de cartera baja }\end{array}$ & $5 \%$ & 1 & 0,05 \\
\hline 2. & $\begin{array}{l}\text { Cambios } \\
\text { administrativos }\end{array}$ & $5 \%$ & 1 & 0,05 \\
\hline 3. & $\begin{array}{l}\text { Devolución de } \\
\text { ventas }\end{array}$ & $10 \%$ & 2 & 0,2 \\
\hline 4. & \begin{tabular}{|l|}
$\begin{array}{l}\text { Equipamiento } \\
\text { viejo }\end{array}$ \\
\end{tabular} & $10 \%$ & 2 & 0,2 \\
\hline 5. & \begin{tabular}{|l|} 
Rotación de \\
personal
\end{tabular} & $20 \%$ & 2 & 0,4 \\
\hline \multicolumn{2}{|l|}{ Total } & $100 \%$ & & 2,75 \\
\hline & \begin{tabular}{|l|} 
Factores \\
externos
\end{tabular} & Ponderación & Clasificación & Valor \\
\hline
\end{tabular}




\begin{tabular}{|c|c|c|c|c|}
\hline Oportunidades & & & & \\
\hline 1. & $\begin{array}{l}\text { Plataformas } \\
\text { online }\end{array}$ & $10 \%$ & 4 & 0,4 \\
\hline 2. & $\begin{array}{l}\text { Adquisición de } \\
\text { nuevo producto }\end{array}$ & $10 \%$ & 3 & 0,3 \\
\hline 3. & $\begin{array}{l}\text { Creación de } \\
\text { nuevo puestos }\end{array}$ & $10 \%$ & 1 & 0,1 \\
\hline 4. & $\begin{array}{l}\text { Espacios con } \\
\text { alta rentabilidad }\end{array}$ & $5 \%$ & 3 & 0,15 \\
\hline 5. & $\begin{array}{l}\text { Creación de } \\
\text { nuevas } \\
\text { tecnologías }\end{array}$ & $15 \%$ & 4 & 0,6 \\
\hline Amenazas & & & & 0 \\
\hline 1. & $\begin{array}{l}\text { Alta } \\
\text { competitividad }\end{array}$ & $12 \%$ & 3 & 0,36 \\
\hline 2. & $\begin{array}{l}\text { Nuevos } \\
\text { impuestos a las } \\
\text { importaciones }\end{array}$ & $8 \%$ & 3 & 0,24 \\
\hline 3. & $\begin{array}{l}\text { Productos } \\
\text { sustitutivos }\end{array}$ & $12 \%$ & 4 & 0,48 \\
\hline 4. & $\begin{array}{l}\text { Cambios en las } \\
\text { necesidades del } \\
\text { consumidor }\end{array}$ & $10 \%$ & 1 & 0,1 \\
\hline 5. & $\begin{array}{l}\text { Devaluación de } \\
\text { productos }\end{array}$ & $8 \%$ & 3 & 0,24 \\
\hline Total & & $100 \%$ & & 2,97 \\
\hline
\end{tabular}

Elaboración: Los autores.

\section{Políticas y estrategias de calidad}

La definición que abarca la palabra política de la calidad son lineamientos establecidos por la empresa, compromisos basados en la formulación de estrategias claras y definidas, en la propuesta que se presenta en este análisis se focaliza en el servicio de calidad que incremente el trato justo y merecido al cliente para una fidelización con la empresa, adicional las estrategias en fuerza de ventas estableciendo una estructura definida donde el consumidor obtenga un abanico de opciones sin dejar de lado la obtención adecuada 
de un financiamiento justo y acorde sus prioridades, pero con los parámetros establecidos por la empresa para no tomar riesgos en la recuperación del capital a continuación se presenta una tabla de los lineamientos en políticas y estrategias.

Tabla 3.

Esquema de políticas y estrategias enfocadas en la calidad.

\begin{tabular}{|c|c|c|}
\hline Políticas & Estrategias & Resultado \\
\hline \multirow{4}{*}{ Calidad } & $\begin{array}{l}\text { Mayor y mejor productividad abarcando } \\
\text { una calidad constante }\end{array}$ & \multirow{4}{*}{$\begin{array}{l}\text { Lograr un buen servicio, } \\
\text { establecer una } \\
\text { fidelización con e } \\
\text { cliente y obtener } \\
\text { beneficios para la } \\
\text { empresa con la finalidao } \\
\text { de posesionarse como } \\
\text { líder en el mercado. }\end{array}$} \\
\hline & Inspección de materia prima & \\
\hline & $\begin{array}{l}\text { Crear planes de capacitación y formación } \\
\text { al personal }\end{array}$ & \\
\hline & Evaluación de desempeño & \\
\hline \multirow{4}{*}{ Ventas } & $\begin{array}{l}\text { Colocar precios en los productos por } \\
\text { temporada alta / baja rotación / falla } \\
\text { estética/ tipo B }\end{array}$ & \multirow{4}{*}{$\begin{array}{l}\text { Establecer procesos } \\
\text { que definan la estructura } \\
\text { de la fuerza de ventas }\end{array}$} \\
\hline & $\begin{array}{l}\text { Diseñar y crear promociones /páginas } \\
\text { web/ redes sociales /pancartas } \\
\text { /promociones / }\end{array}$ & \\
\hline & $\begin{array}{l}\text { Establecer comisiones de ventas por } \\
\text { objetivos cumplidos en producto y/o } \\
\text { servicios }\end{array}$ & \\
\hline & $\begin{array}{l}\text { Conformar un registro de proveedores } \\
\text { calificados/ mantener un stock óptimo de } \\
\text { productos/ controlar el abastecimiento a } \\
\text { los almacenes }\end{array}$ & \\
\hline \multirow{3}{*}{ Créditos } & Revisión del historial crediticio & \multirow{3}{*}{$\begin{array}{lr}\text { Incrementar } & \text { objetivos } \\
\text { financieros, maximizar } \\
\text { las ventas seguras para } \\
\text { minimizar ra }\end{array}$} \\
\hline & Análisis de la capacidad de pago & \\
\hline & & \\
\hline
\end{tabular}


Establecer un monto de crédito /tiempo de financiamiento /tazas de interés Verificación de campo (ubicación domiciliaría) referencias bancarias / garantías

Elaboración: Los autores.

\section{Ejecución}

La ejecución es el factor primordial de la empresa, para que los resultados vayan acorde a un diseño planteado, el personal juega un rol importante que es de alinear los esfuerzos individuales conjuntamente con las estrategias, esta hará la diferencia entre las empresas del mercado requiriendo disciplina y organización, se planteó la matriz QFD (despliegue de la función de calidad) donde se establece las prioridades en ejecutar las estrategias para verificar su relación con las funciones del personal o encargados de las áreas que se estudian, adicional se presenta la relación entre las áreas que debe trabajar conjuntamente y se establece un análisis cuantitativo de competencias con las principales empresas de electrodomésticos para identificar como se encuentra la compañía en la actualidad como se presenta en la tabla adjunta. 


\section{Tabla 4.}

Análisis de la función de calidad.

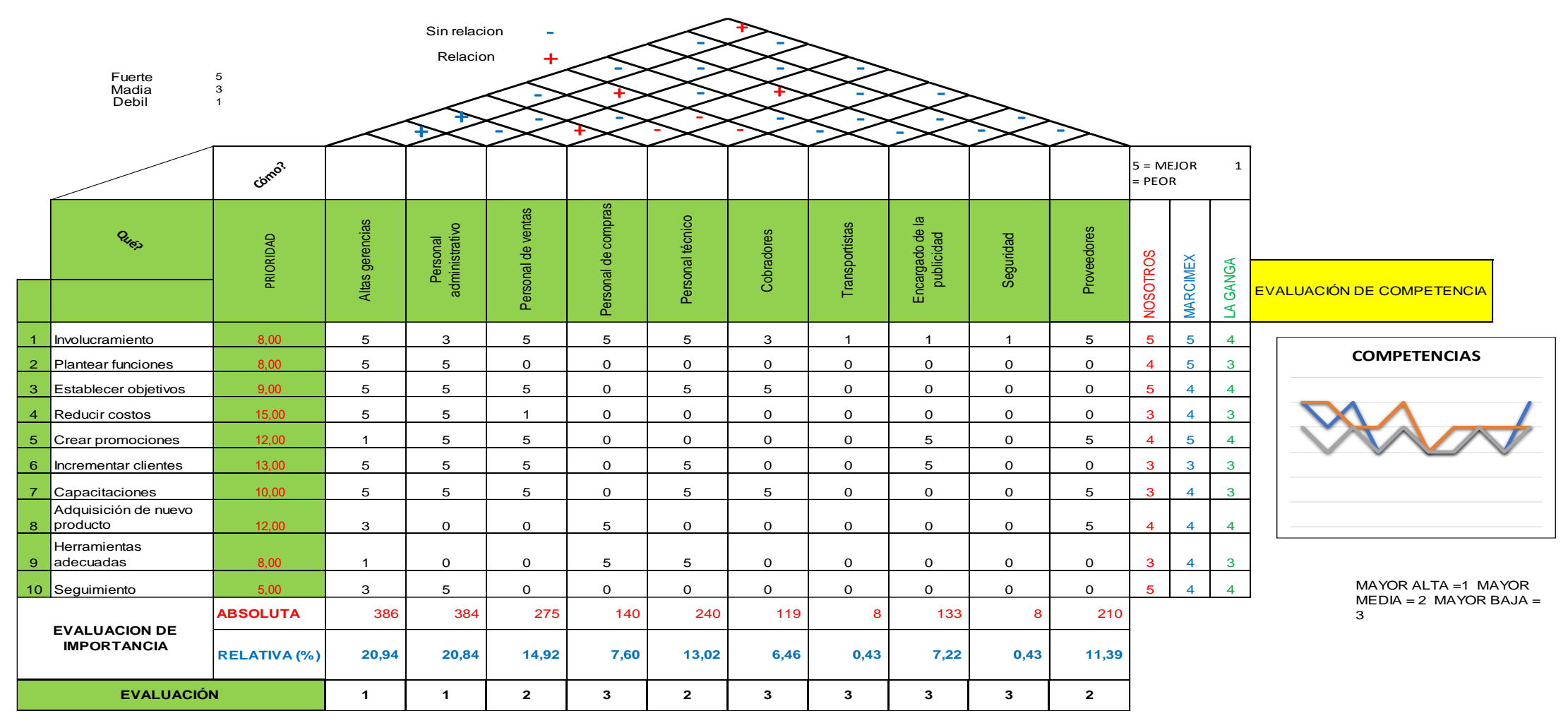


Revista Arbitrada Interdisciplinaria KOINONIA

Año VI. Vol VI. N 12. Julio - Diciembre. 2021

Hecho el depósito de Ley: FA2016000010

ISSN: 2542-3088

FUNDACIÓN KOINONIA (F.K). Santa Ana de Coro. Venezuela.

María Belén Jarrín-Arias; Jorge Edwin Ormaza-Andrade

\section{Mejoramiento continúa}

Para idear un plan eficaz se necesita considerar varios puntos, en la mayoría es importante entender que estas estrategias planteadas parar mejora se aplican constantemente.

En primera instancia hay que detectar las causas que provocan que los procesos de calidad, sé estanque por factores que no se visualizan si no es a través de un análisis de variabilidad donde se detectan las falencias, llevando así a ser consideradas para el seguimiento respectivo con la finalidad de ir mitigando el efecto que se representa en el cuadro adjunto. 
Revista Arbitrada Interdisciplinaria KOINONIA

Año VI. Vol VI. N 12. Julio - Diciembre. 2021

Hecho el depósito de Ley: FA2016000010

ISSN: 2542-3088

FUNDACIÓN KOINONIA (F.K). Santa Ana de Coro. Venezuela.

María Belén Jarrín-Arias; Jorge Edwin Ormaza-Andrade

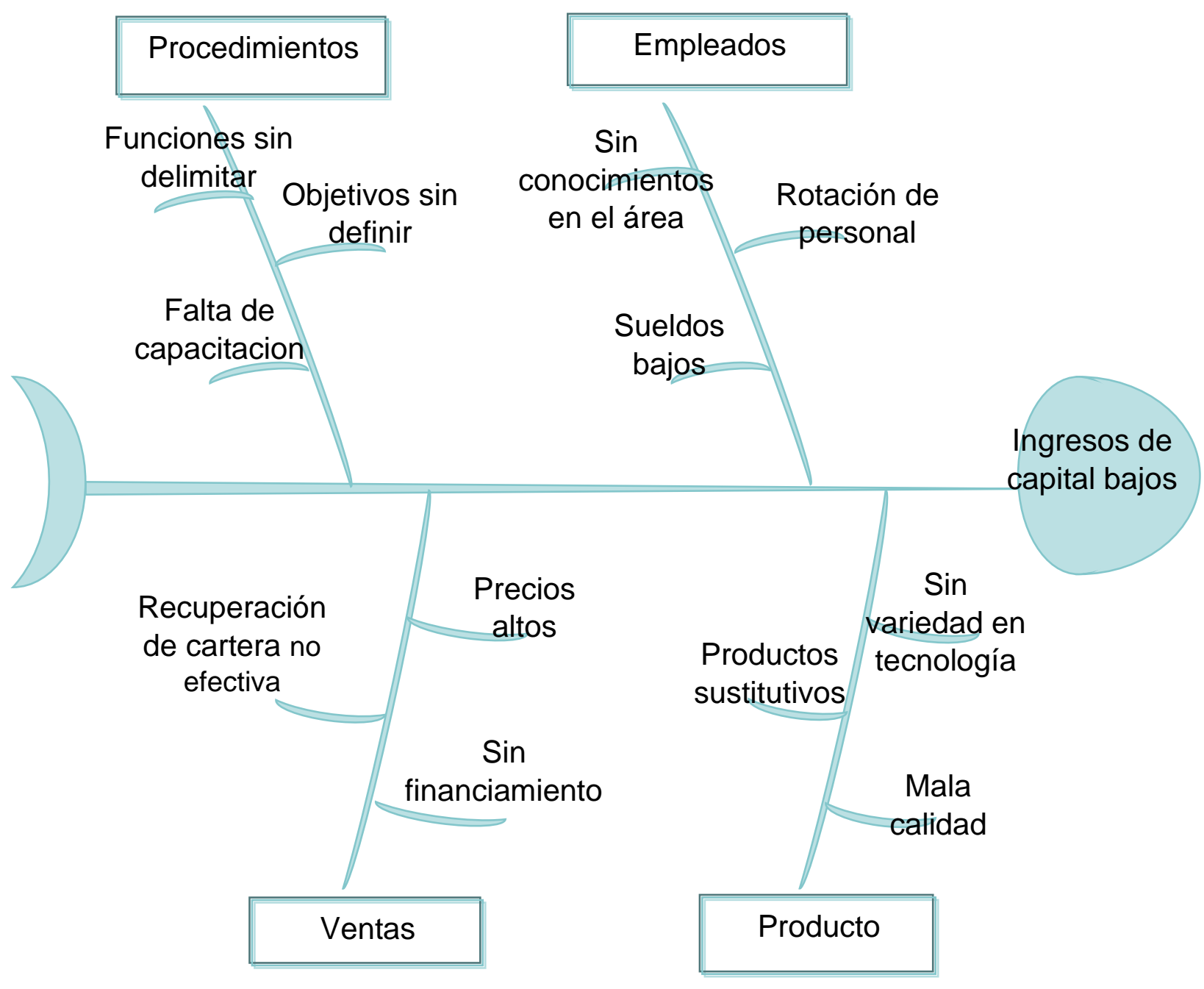

Figura 2. Análisis de variabilidad en las empresas de electrodomésticos.

Fuente: Bonet-Borjas, (2005). 


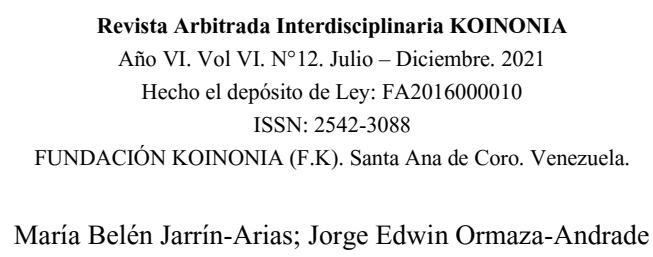

\section{CONCLUSIONES}

En la actualidad la gestión de calidad es el servicio que toda empresa debe incluir sin importar el ámbito, es prioridad en la identificación de su nombre y como punto principal se debe identificar su falencia en el servicio con el cliente e incluir varios procedimientos meticulosos que llevan a la prestación de un servicio con calidad, de este modo se obtiene un valor agregado que genera satisfacción al consumidor.

En la investigación se determinó que en las empresas de electrodomésticos deben profundizar en una información clara en el empleado ya que no cuentan con esa herramienta para llenar las expectativas del cliente, esto es debido a la rotación del personal en el área de ventas y a la demanda acelerada de modelos en producción.

Es importante una respuesta rápida al cliente sobre el tiempo en cubrir una garantía sea esta reparación o cambio de producto y /o servicio, ya que genera insatisfacción en el consumidor llevando a una publicidad negativa hacia la empresa.

Adicional se determinó que el factor externo es un contrapié de las empresas, ocasionando atrasos de los pagos, productos con devolución y bajas ventas por cuestión de la economía actual del país

Es indispensable proceder con la ejecución de un plan de estrategias de manera inmediata y el seguimiento del mismo con la finalidad de que el personal administrativo y técnico se involucre directamente en alcanzar los objetivos para una mejor la situación empresarial.

\section{FINANCIAMIENTO}

No monetario.

\section{AGRADECIMIENTO}

A la Jefatura de Posgrados de la Universidad Católica de Cuenca por su apoyo a la investigación. 


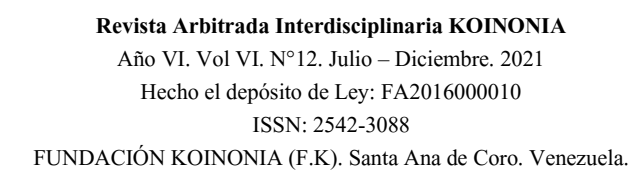

María Belén Jarrín-Arias; Jorge Edwin Ormaza-Andrade

\section{REFERENCIAS CONSULTADAS}

Alcívar-Arízaga, C., Erazo Álvarez, J., \& Narváez Zurita, C. (2019). El reto de la gestión de calidad en las IES públicas del Ecuador Caso: Universidad de Cuenca [The challenge of quality management in public HEls in Ecuador Case: University of Cuenca]. Revista Arbitrada Interdisciplinaria Koinonía, 4(1), 97-124. http://dx.doi.org/10.35381/r.k.v4i1.373

Antúnez-Saiz, V. (2016). Sistemas integrados de gestión: de la teoría a la práctica empresarial en Cuba [Integrated Management Systems: from the Theory to the Practice in the Enterprise System in Cuba]. Cofin Habana, 10(2), 1-28.

Baldrige National Quality Program USA. (2006). Modelo de Excelencia en la Gestión Malcolm Baldrige [Malcolm Baldrige Model of Excellence in Management]. https://n9.cl/irp0c

Bernal, J. (2013). El círculo de Deming mejora continúa [Deming's circle continues to improve]. Obtenido de https://n9.cl/wemn

Bodnar, K. (2020). Datos clave: cómo COVID-19 afecta el rendimiento de ventas y marketing [Key Facts: How COVID-19 Affects Sales and Marketing Performance]. Obtenido de https://n9.cl/te0l1

Bonet-Borjas, C. (2005). Ley de Pareto aplicada a la fiabilidad [Pareto's law applied to reliability]. Ingeniería Mecánica, 8(3),1-9.

Bonilla-Rivera, L. M., \& Paraguay-Alvarado, A. L. (2017). Estudio de competitividad de la industria ecuatoriana de electrodomésticos de línea blanca"proyecto de titulación [Study of the competitiveness of the Ecuadorian white goods industry "degree project]. Escuela Superior Politecnica Del Litoral, Guayaquil. Obtenido de https://n9.cl/kdf81

Delgado-Estrada, S, Villacis Aveiga, W, \& Chávez Garcés, A. (2018). Análisis del comportamiento de compra en servicios intangibles [Analysis of purchasing behavior in intangible services]. Revista Universidad y Sociedad, 10(3), 125-133.

ISO 9001. (2020). ¿Qué es la gestión de la calidad? [What is quality management?]. https://n9.cl/hlvkk 


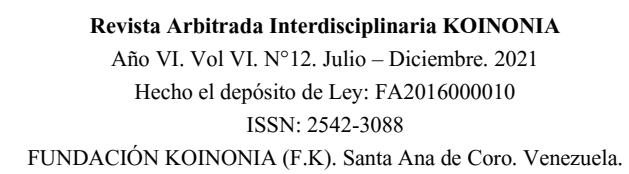

María Belén Jarrín-Arias; Jorge Edwin Ormaza-Andrade

León-Ramentol, C, Menéndez-Cabezas, A, Rodríguez-Socarrás, I, López-Estrada, B, García-González, M, \& Fernández-Torres, S. (2018). Importancia de un sistema de gestión de la calidad en la Universidad de Ciencias Médicas [Importance of a quality management system at the university of medical sciences]. Revista Archivo Médico de Camagüey, 22(6), 843-857.

Martínez, B. (2008). Calidad. ¿Qué es el modelo EFQM (European Foundation for Quality Management)? Anales de pediatría continuada, 6(5), 313-318. https://doi.org/10.1016/S1696-2818(08)74887-X

Medina-León, A, Nogueira Rivera, D, Hernández-Nariño, A, \& Comas Rodríguez, R. (2019). Procedimiento para la gestión por procesos: métodos y herramientas de apoyo [Procedure for process management: methods and support tools]. Ingeniare. Revista chilena de ingeniería, 27(2), 328342. https://dx.doi.org/10.4067/S0718-33052019000200328

Montero-Tapia, I. F., Erazo Álvarez, J. C., Narváez Zurita, C. I., \& Ormaza Andrade, J. E. (2019). Desarrollo organizacional como estrategia de modernización de la calidad del servicio de alimentos y bebidas [Organizational development as a strategy to modernize the quality of the food and beverage service]. Visionario Digital, 3(2.2), 103-119. https://doi.org/10.33262/visionariodigital.v3i2.2.618

Palmira-Lopez, F. (2010). Gestión eficaz de reclamaciones [Effective claims management]. España: Netbiblo, S.L

Perez-Montero, O. I. (2013). Implementación de un sistema de gestión de calidad según la norma ISO 9001 - 2000, en la compañía de electrodomésticos famcev s.a., de la ciudad de Riobamba, provincia de Chimborazo, período 2012 - 2016 [Implementation of a quality management system according to the ISO $9001-2000$ standard, in the household appliances company famcev s.a., of the city of Riobamba, province of Chimborazo, period 2012 - 2016]. https://n9.cl/wdyid

Romero-Bermúdez, E, \& Díaz-Camacho, J. (2010). El uso del diagrama causa-efecto en el análisis de casos [The use of the cause-effect diagram in case analysis]. Revista Latinoamericana de Estudios Educativos (México), XL(3-4),127-142. 


\author{
Revista Arbitrada Interdisciplinaria KOINONIA \\ Año VI. Vol VI. N¹2. Julio - Diciembre. 2021 \\ Hecho el depósito de Ley: FA2016000010 \\ ISSN: 2542-3088 \\ FUNDACIÓN KOINONIA (F.K). Santa Ana de Coro. Venezuela. \\ María Belén Jarrín-Arias; Jorge Edwin Ormaza-Andrade
}

Silva-Treviño, J, Macías-Hernández, B, Tello-Leal, E, \& Delgado-Rivas, J. (2021). La relación entre la calidad en el servicio, satisfacción del cliente y lealtad del cliente: un estudio de caso de una empresa comercial en México [The relationship between service quality, customer satisfaction, and customer loyalty: A case study of a trading company in Mexico]. CienciaUAT, 15(2), 85-101. https://doi.org/10.29059/cienciauat.v15i2.1369

(C2021 por los autores. Este artículo es de acceso abierto y distribuido según los términos y condiciones de la licencia Creative Commons Atribución-NoComercial-Compartirlgual 4.0 Internacional (CC BY-NC-SA 4.0)

(https://creativecommons.org/licenses/by-nc-sa/4.0/). 